\title{
A New Strategy of Desktop Sharing Mechanism Based on Mixed Mode
}

\author{
Long Zhang ${ }^{1,}$ a , Yaling Yin ${ }^{2, b}$ \\ ${ }^{1}$ Xi'an Eurasia University, Xi'an, Shaanxi, 710065, China \\ ${ }^{2}$ Xi'an University of Finance and Economics, Information School, 710100, China \\ adir_zl@qq.com,byyll@126.com
}

Keywords: video conferencing; desktop sharing; mixed mode; distributed; centralized.

\begin{abstract}
As an important strategy of video communication, desktop sharing is widely used in video conference communication. However, high consumption of CPU problems, bandwidth costs, are still the key to the desktop sharing. Based on the improvement of CPU utilization, reduce the requirement of network bandwidth; the quality of video communication is improved. In this paper, a hybrid mode selection strategy of distributed and centralized is proposed, and a small scale test is conducted.
\end{abstract}

\section{Introduction}

Currently, the video conferencing system usually provided desktop sharing, application sharing function. And the desktop sharing client obtained screen information by hook technology, through the designated encoding technology, compressed data transmission to the conference server or other participant's client. If the conference server mode is adopted, the server sends the information to other participants through the multicast for display. A vast amount of independent literature were purposed and there were some optimization methods and techniques in the academic and technical fields, such as compiling and decoding algorithms with high compression ratio and region matching transmission algorithm. Many scholars had adopted the method of deploying proxy server to satisfy high concurrency capacity [1]. But these technologies or methods must sacrifice CPU usage in exchange for reducing bandwidth. Although to some extent to solve some problems, it can exposed many shortcomings, such as high compression ratio encoding and decoding technology would bring higher CPU consumption to the client, and the proxy server mode would improve hardware costs and bandwidth costs. How to find a reasonable solution between the scale and cost of services has always been a problem that the academic circles and technical circles try to explore.

\section{Realization Strategy}

\subsection{Key Technology}

According to the firewall rules and business requirements of both communication mode using distributed or centralized mode of communication, the key point is sorting technology, the sorting algorithm based on the difficulty of cracking Firewall and business requirements, and then the weighted summation, classified according to the score, 1 is distributed, 2 is centralized. Figure 1 is a desktop shared system architecture diagram, in which desktop sharers and observers can be based on the type of the NAT (Symmetric / Cone) and firewall rules (to allow / ban UDP packet), select UDP or TCP protocol for communication [2]. When using UDP protocol, ICE server is responsible for P2P tunneling and transparent UDP packet forwarding; using TCP protocol, Refelctor server is responsible for the initiative to read the desktop information from the sharing client, and then according to the observer's bandwidth adaptively adjust to synchronize desktop content backwardness to observer. The client can automatically select the appropriate working mode according to the actual situation of NAT / Firewall [3]. In addition, the system allows both distributed and centralized working mode of coexistence. 


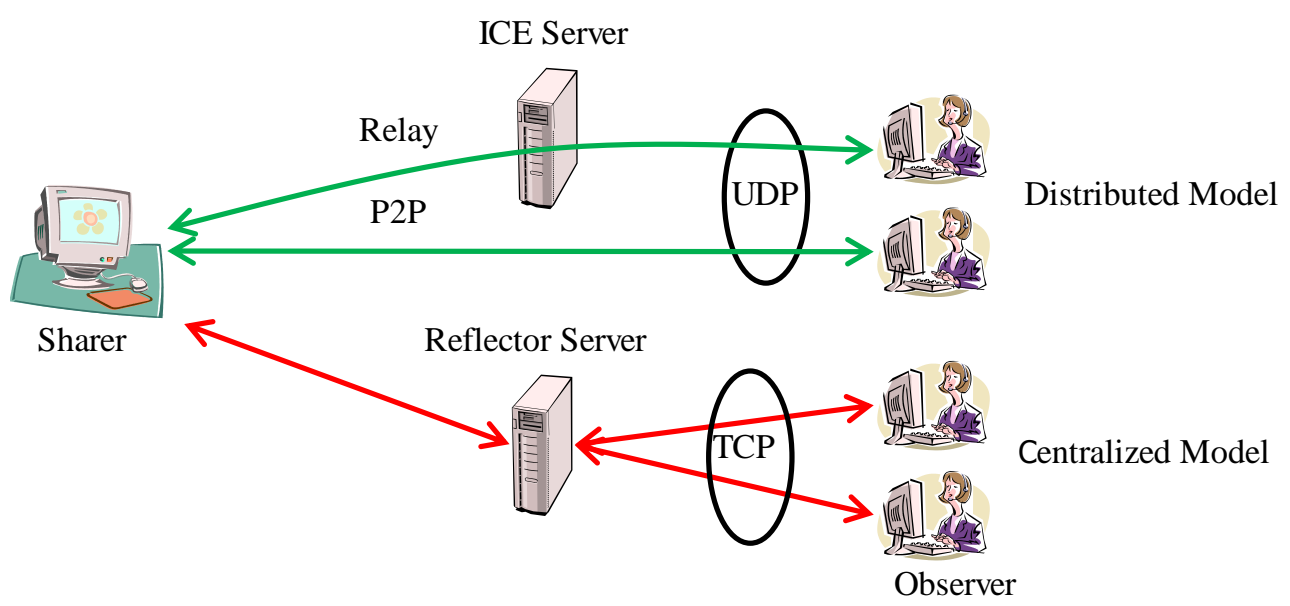

\subsection{Architecture Function Module}

Fig. 1 The system architecture

(1) ICE (Internet Connectivity Establishment, that is, interactive connection establishment) protocol processing module

ICE protocol module is mainly used to implement $\mathrm{P} 2 \mathrm{P}$ (peer-to-peer) connection between clients [4]. Through the use of multiple protocols and network connection mechanisms such as STUN (Simple Traversal of UDP through NATs - STUN), TURN (ITRS), ICE can know the network structure of the client and the network addresses that these devices can communicate with The basic process of ICE consists of two steps. The first step is to collect all types of addresses. The second step is to pair the local and remote addresses, and sort, and then perform the connectivity check.

(2) Reflector multicast processing module

Reflector multicast processing module is mainly to complete the multicast function of information, desktop sharer by reverse connection to the Reflector module, Reflector acts as the role of observer, that is, getting the information from the sharer, while other observers are also connected to Reflector and request information, Reflector according to each observer's coding, decoding capabilities and the contents of the request one by one forwarded to share the information to complete the purpose of desktop sharing.

(3) Pub / Sub (publishing / subscription) protocol processing module

The Pub / Sub protocol processing module is a multicast service that cooperates with the Reflector multicast processing module to complete the desktop information on specific occasions [5]. The desktop sharing party creates the conference theme and other observers subscribe to the topic to complete the forwarding of the information to the specific conference. In this way, Reflector multicast processing module can serve multiple purposes simultaneously.

\subsection{Realization}

System is divided into client and server two roles of two parts. The following describes the desktop sharing function respectively the basic flow on the client and the server:

(1) Client

Step 1: Obtain the server configuration. After the client starts the desktop sharing function, it will first send the address information of the server to the module through the desktop sharing module setting interface, including the address of the ICE and Refelctor servers and the protocols used;

Step 2: Log in to the ICE server. Desktop sharing landing module interfaceTry to log in ICE server. If the login fails, it indicates that the user's firewall rules may block UDP packets. Therefore, only the centralized mode can be used. Thereby proceeding to step 3. If the landing is successful, you can use a distributed model or centralized mode. This depends on the firewall rules between two communication parties and business requirements;

Step 3: Login Reflector server, the client login interface through the TCP mode to connect to the Reflector server; 
Step 4: Start the share, and the client creates the desktop share through the desktop sharing module initiator interface, and broadcasts the message to all viewers. The content of the message can be divided into two kinds. If the sharer supports the distributed mode, the message includes The local address, NAT address and Relay address. If the sharer does not support the distributed mode, the message is the reflector server address;

Step 5: Start the observer. Observers were received after the broadcast of the sharer, first call the desktop sharing boot interface module in order to start the observer desktop sharing module, then according to the participants and observers of the firewall to automatically select the appropriate working mode;

Step 6: End sharing. When the sharer or the observers are stopped to share, can be proactively closed with the peer or Reflector server connection, which once all the participants end of the desktop sharing service, Reflector to end the conference sessions, Release resources;

(2) Server

Step 1: Start the ICE server. Real time is receiving and processing client-side request to help the client to complete the work through the firewall;

Step 2: Starts the Relector server to listen and process client connection requests in real time. Where sharer or the observer, after successfully connecting the Reflector server, first send a request to the server to create or subscribe to the VNC session. Reflector server according to the above information, all participants can be organized into the same VNC session, and by number and type to distinguish between the identities of the participants.

\section{Experimental Application and Comparison}

Based on the method proposed in this paper, we designed the RK-VC (Rongke Video Teleconferencing System). First start the ICE server, real-time receiving and processing client-side request, and then start the Relector server, real-time monitoring and processing client connection request, waiting for the share or observer connection. Then the client or observer of the client enters the service address in the browser to $\log$ on the connection. The client login interface connects to the Reflector server through the TCP mode. The sharing client creates the desktop share through the desktop sharing module start interface and broadcast to all observers via a message. After receiving the message, the observer first invokes the desktop sharing module observer to start the interface to start the observer desktop sharing module, and then automatically selects the appropriate working mode according to the firewall conditions of both the sharer and the observer.

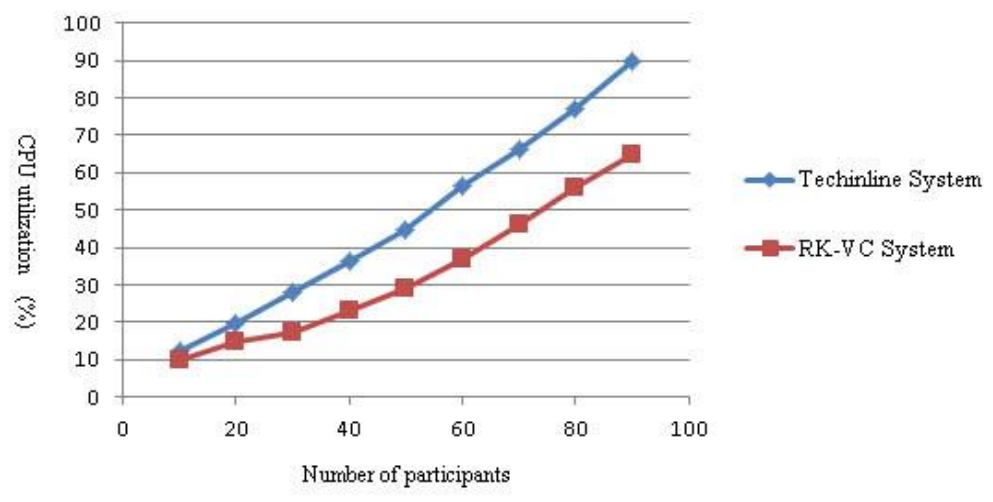

Fig.2 Comparison of CPU occupancy rate on $1 \mathrm{Mb}$ bandwidth 


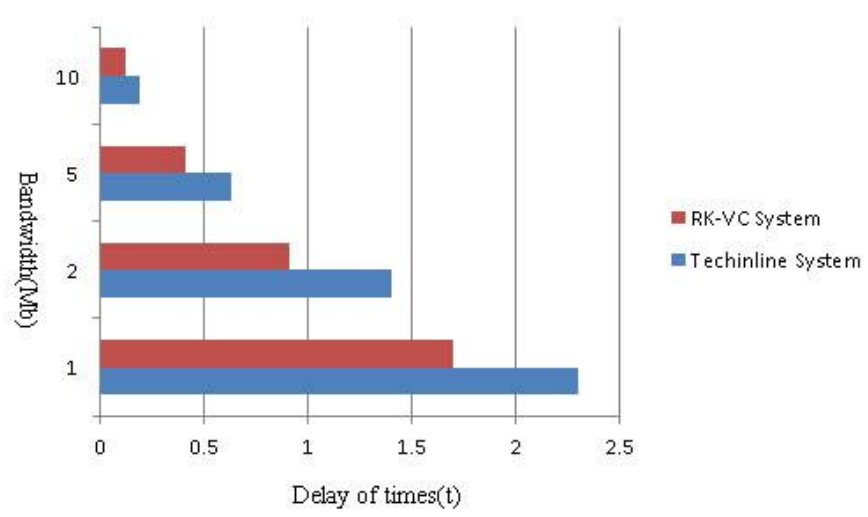

Fig. 3 Comparison of document sharing delay

In the Figure 2, we show the result of using the TechInline document sharing system and RK-VC system to test in $1 \mathrm{Mb}$ bandwidth. With the increase of the number of participants, the occupancy rate of the CPU is increasing. We can see that CPU occupancy rate of RK-VC system is smaller.

In Figure 3, we used the TechInline document sharing system and the RK-VC system to share a $200 \mathrm{k}$ desktop document to test. The results show that the RK-VC system uses less time to share documents under the same bandwidth.

At present, the realization of audio / video interaction, text communication, document sharing and other desktop sharing means of interaction. Through the practical application test, the desktop sharing mechanism based on mixed mode can effectively improve the utilization of CPU and improve the communication effect when the remote desktop sharing interaction under limited bandwidth.

\section{Conclusion}

In this paper, we propose a hybrid mode selection strategy, which is distributed and centralized. There are two main characteristics:

1) Through the publication of the subscription model to solve a single server for multiple conferencing services at the same time, through the server multicast greatly reduces the share of the client's bandwidth consumption and CPU pressure.

2) The design allows distributed and centralized desktop sharing of the two modes of coexistence, according to bandwidth, server hardware conditions to adaptively choose the appropriate desktop sharing working mode, thus ensuring limited hardware resources for large scale desktop sharing services, but also allows flexible configuration according to the use of scenarios.

\section{References}

[1]. Zhao W, Huang F, Bu S, et al. Performance Evaluation of Concurrent-access-intensive WebGIS Applications Based on CloudStack[J]. International Journal of Advancements in Computing Technology, 2014, 6(5): 41.

[2]. Fall K R, Stevens W R. TCP/IP illustrated, volume 1: The protocols [M]. addison-Wesley, 2011.

[3]. Paganelli F, Ulema M, Martini B. Context-aware service composition and delivery in NGSONs over SDN [J]. IEEE Communications Magazine, 2014, 52(8): 97-105.

[4]. $\mathrm{Xu} \mathrm{Z}$, Chen $\mathrm{L}, \mathrm{Gu} \mathrm{G}$, et al. PeerPress: utilizing enemies' P2P strength against them[C]/Proceedings of the 2012 ACM conference on Computer and communications security. ACM, 2012: 581-592.

[5]. Sibert W O. Illumination control network: U.S. Patent Application 13/243,449[P]. 2012-1-19. 